\title{
GLOBAL ATTRACTIVITY IN A FOUR-TERIM RECURRENCE RELATION
}

\author{
LONG-TU LI AND SUI SUN CHENG
}

\begin{abstract}
Positive solutions of the four-term recurrence relation $x_{n+1}=f\left(x_{n}\right) g\left(x_{n-1}, x_{n-2}\right)$ are shown to converge to its positive equalibrium points under relative mild conditions.
\end{abstract}

\section{Introduction}

The asymptotic behavior of sequences defined by recurrence relations has long been discussed in various branch of sciences (see e.g. Kocic and Ladas [1]). In this note, we are concerned with the asymptotic behavior of the set $\Omega$ of real sequences $\left\{x_{n}\right\}_{n=-2}^{\infty}$ defined by $x_{-2}=\alpha>0, x_{-1}=\beta>0, x_{0}=\gamma>0$ and

$$
x_{n+1}=f\left(x_{n}\right) g\left(x_{n-1}, x_{n-2}\right), \quad n=0,1,2, \ldots,
$$

where

(H1) $f:(0, \infty) \rightarrow R$ and $g:[0, \infty) \times[0, \infty) \rightarrow R$ are positive functions; and

(H2) $f$ is nondecreasing and $g$ is nonincreasing in each of its independent variables.

A positive fixed point $x^{*}$ that satisfies $x=f(x) g(x, x)$ is also called a positive equilibrium point of equation (1.1). Our objective of this note is to show that under mild conditions on the functions $f$ and $g$, every real sequence in $\Omega$ tends to one of the positive equilibrium points of (1.1).

Similar results have been obtainied for a number of recureence relations, see e.g. Kocic and Ladas [1], Camouzis et al. [2], Li et al. [3], and Li [4]. Indeed, this note is motivated by a concern raised in Kocic and Ladas $[1$, p.46] related to the stability of recurrence relations.

\section{Stability Criteria}

We need to establish a boundedness criteria as a preparatory result for proving our assertion. Its proof is motivated by that of Theorem 4.1 in [2], and involves novel use of a polynomial equation.

Received February 2, 1999.

1991 Mathematics Subject Classification. 39A10.

Key words and phrases. Equilibrium point, four-term recurrence relation, attractivity, difference equation. 
Theorem 1. Assume that (H1) and (H2)hold. Suppose further that there are positive constants $A, B, L, p, q$ and $r$ such that

$$
\begin{array}{r}
f(x) \leq A x^{p}, \quad x \geq L>0 \\
g(x, y) \leq \frac{B}{x^{q} y^{r}}, \quad x, y \geq L>0
\end{array}
$$

and

$$
\lambda^{3}-p \lambda^{2}+q \lambda+r=0
$$

does not have any positive roots. Then every sequence in $\Omega$ is bounded.

Proof. Let $\left\{\lambda_{n}\right\}_{n=0}^{\infty}$ be defined by $\lambda_{0}=p$ and

$$
\lambda_{n}=p-\frac{\lambda_{n-1} q+r}{\lambda_{n-1}^{2}}, \quad n=1,2,3, \ldots
$$

We assert that there is some positive integer $N$ such that $\lambda_{0}, \lambda_{1}, \ldots, \lambda_{N-1}>0$ and $\lambda_{N} \leq 0$. Indeed, if $p^{3} \leq p q+r$, then

$$
\lambda_{1}=p-\frac{p q+r}{p^{2}}=\frac{p^{3}-(p q+r)}{p^{2}} \leq 0 .
$$

If $p^{3}>p q+r$, then

$$
\lambda_{1}=\frac{p^{3}-(p q+r)}{p^{2}}>0,
$$

and

$$
\lambda_{1}-\lambda_{0}=-\frac{p q+r}{p^{2}}<0 .
$$

If $\lambda_{n}>0$ for all $n$, then since

$$
\lambda_{n+1}-\lambda_{n}=\left(\lambda_{n}-\lambda_{n-1}\right) \frac{\lambda_{n} \lambda_{n-1} q+\lambda_{n} r+\lambda_{n-1} r}{\lambda_{n}^{2} \lambda_{n-1}^{2}}
$$

for $n \geq 1$, we easily see by induction that $\left\{\lambda_{n}\right\}$ decreases to a nonnegative limit $\lambda$. By taking limits on both sides of (2.2), we see that $\lambda$ is a nonnegative root of (2.1), furthermore, it must be positive, since 0 is not a root of (2.1). However, by our assumptions, the above equation does not have any nonnegative solution. This is a contradiction. In other words, there is some positive integer $N$ such that $\lambda_{0}, \lambda_{1}, \lambda_{2}, \ldots, \lambda_{N-1}>0$ and $\lambda_{N} \leq 0$.

Let $\left\{x_{n}\right\}_{n=-2}^{\infty}$ be a real sequence in $\Omega$. If $f$ is bounded by $M$, then clearly

$$
x_{n+1}=f\left(x_{n}\right) g\left(x_{n-1}, x_{n-2}\right) \leq M g(0,0)
$$

for all $n \geq 0$. If $f$ is not bounded, then $\lim _{n \rightarrow \infty} f\left(x_{n}\right)=\infty$ since $f$ is nondecreasing. If $\left\{x_{n}\right\}$ is not bounded above, then there is a subsequence $\left\{x_{n_{i}}\right\}_{i=0}^{\infty}$ such that $\lim _{i \rightarrow \infty} x_{n_{i}}=$ 
$\infty$. Without loss of any generality, we may also assume that $n_{i+1}-n_{i}>2 N+3$. Since $g(x, y)$ is decreasing in $x$ and in $y$, thus

$$
x_{n_{i}}=f\left(x_{n_{i}-1}\right) g\left(x_{n_{i}-2}, x_{n_{i}-3}\right) \leq f\left(x_{n_{i}}\right) g(0,0), \quad i=0,1,2, \ldots,
$$

which shows that $\lim _{i \rightarrow \infty} f\left(x_{n_{i}-1}\right)=\infty$. But then $\lim _{i \rightarrow \infty} x_{n_{i}-1}=\infty$. Similarly, we may show that

$$
\lim _{i \rightarrow \infty} x_{n_{i}-k}=\infty, \quad k=1,2, \ldots, 2 N+3
$$

As a consequence,

$$
\lim _{i \rightarrow \infty} \frac{x_{n_{i}}}{x_{n_{i}-1}^{\lambda_{N}}}=\infty
$$

On the other hand, let $M$ be a positive integer such that

$$
x_{n_{i}-k} \geq L, \quad k=0,1,2, \ldots, 2 N+3,
$$

for $i \geq M$. Since

$$
x_{n_{i}-k}=f\left(x_{n_{i}-k-1}\right) g\left(x_{n_{i}-k-2}, x_{n_{i}-k-3}\right) \leq A B \frac{x_{n_{i}-k-1}^{p}}{x_{n_{i}-k-2}^{q} x_{n_{i}-k-3}^{r}},
$$

thus

$$
\lim _{i \rightarrow \infty} \frac{x_{n_{i}-k}}{x_{n_{i}-k-1}^{p}}=0
$$

for $k=0,1,2, \ldots, 2 N$ and

$$
\frac{x_{n_{i}-k}}{x_{n_{i}-k-1}^{\lambda_{j}}} \leq A B \frac{x_{n_{i}-k-1}^{p-\lambda_{j}}}{x_{n_{i}-k-2}^{q} x_{n_{i}-k-3}^{r}}=A B\left(\frac{x_{n_{i}-k-1}}{x_{n_{i}-k-2}^{\lambda_{j}-1}}\right)^{p-\lambda_{j}}\left(\frac{x_{n_{i}-k-2}}{x_{n_{i}-k-3}^{\lambda_{j}-1}}\right)^{r / \lambda_{j}-1}
$$

for $k=0,1,2, \ldots, 2 N, j=0,1,2, \ldots, N-1$ and $i \geq M$, where we have used the fact that $\lambda_{j-1} q=\lambda_{j-1}^{2} p-\lambda_{i-1}^{2} \lambda_{j}-r$ in obtaining the last equality. But then

$$
\begin{aligned}
\frac{x_{n_{i}}}{x_{n_{i}-1}^{\lambda_{N}}} \leq & A B\left(\frac{x_{n_{i}-1}}{x_{n_{i}-2}^{\lambda_{N}-1}}\right)^{p-\lambda_{N}}\left(\frac{x_{n_{i}-2}}{x_{n_{i}-3}^{\lambda_{N}-1}}\right)^{r / \lambda_{N-1}} \\
\leq & (A B)^{1+p-\lambda_{N}+r / \lambda_{N-1}}\left(\frac{x_{n_{i}-2}}{x_{n_{i}-3}^{\lambda_{N-2}}}\right)^{\left(p-\lambda_{N}\right)\left(p-\lambda_{N-1}\right)}\left(\frac{x_{n_{i}-3}}{x_{n_{i}-4}^{\lambda_{N}-2}}\right)^{\left(p-\lambda_{N}\right)\left(r / \lambda_{N-2}\right)} \\
& \times\left(\frac{x_{n_{i}-3}}{x_{n_{i}-4}^{\lambda_{N}-2}}\right)^{\left(p-\lambda_{N-1}\right)\left(r / \lambda_{N-1}\right)}\left(\frac{x_{n_{i}-4}}{x_{n_{i}-5}^{\lambda_{N}-2}}\right)^{\left(r / \lambda_{N-1}\right)\left(r / \lambda_{N-2}\right)} \\
\leq & \cdots \\
\leq & A^{m} B^{m}\left(\frac{x_{n_{i}-N}}{x_{n_{i}-N-1}^{p}}\right)^{s_{1}} \cdots\left(\frac{x_{n_{i}-2 N}}{x_{n_{i}-2 N-1}^{p}}\right)^{s_{2 N}}
\end{aligned}
$$


for some positive numbers $m, s_{1}, \ldots, s_{2 N}$ where $s_{1}, \ldots, s_{2 N}$ are rational expressions of $\lambda_{1}, \lambda_{2}, \ldots, \lambda_{N-1}$. It follows from (2.4) that

$$
\lim _{i \rightarrow \infty} \frac{x_{n_{i}}}{x_{n_{i}-1}^{\lambda_{N}}}=0
$$

which is contrary to (2.3). The proof is complete.

We remark that the polynomial equation (2.1) does not have any positive roots if, and only if, the difference equation

$$
x_{n+3}-p x_{n+2}+q x_{n+1}+r=0, \quad n=0,1,2, \ldots
$$

is oscillatory (see e.g. [1, Theorem 1.2.1]). Furthermore, oscillatory criteria for such difference equations can be found in a number of recent papers.

As an application, let us consider the recurrence relation

$$
y_{n+1}=\frac{e+y_{n}^{2}}{d+y_{n-1} y_{n-2}}, \quad n=0,1,2, \ldots
$$

where $d, e>0$. Here $f(x)=e+x^{2} \leq A x^{2}$ for all large $x$, and $g(x, y)=1 /(d+x y) \leq 1 /(x y)$ for all large $x$ and $y$. Furtheremore, the polynomial

$$
h(\lambda)=\lambda^{3}-2 \lambda^{2}+\lambda+1
$$

does not have any positive roots since $h(0)=1, h(\infty)=\infty$ and $\min _{\lambda>0} h(\lambda)=13>0$. Hence all the assumptions in Theorem 1 are satisfied. This shows that every solution of (2.5) defined by positive initial conditions is bounded. We assert further that the sequence $\left\{y_{n}\right\}$ defined by positive initial conditions and (2.5) converges when $d>1 / 4$. Indeed, by Theorem 1 , the sequence $\left\{y_{n}\right\}$ is bounded between 0 and some positive number $M$. Note that

$$
y_{n+1}=\frac{e+y_{n}^{2}}{d+y_{n-1} y_{n-2}} \geq \frac{e}{d+y_{n-1} y_{n-2}} \geq \frac{e}{d+M^{2}}
$$

thus

$$
\frac{1}{\sqrt{y_{k}}} \leq \sqrt{\frac{d+M^{2}}{e}} \equiv H, \quad k \geq-2 .
$$

On the other hand,

$$
\begin{aligned}
y_{n+1} & =\frac{e+y_{n}^{2}}{d+y_{n-1} y_{n-2}} \\
& =\frac{e}{d+y_{n-1} y_{n-2}}+\frac{y_{n} y_{n}}{d+y_{n-1} y_{n-2}} \\
& =\frac{e}{d+y_{n-1} y_{n-2}}+\frac{y_{n}\left(e+y_{n-1}^{2}\right)}{\left(d+y_{n-1} y_{n-2}\right)\left(d+y_{n-2} y_{n-3}\right)} \\
& =\frac{y_{n} e}{d+y_{n-1} y_{n-2}}+\frac{\left.y_{n-1} y_{n-2}\right)\left(d+y_{n-2} y_{n-3}\right)}{\left(d+y_{n-1}\right.}
\end{aligned}
$$




$$
\begin{aligned}
& +\frac{y_{n} y_{n-1}\left(e+y_{n-2}^{2}\right)}{\left(d+y_{n-1} y_{n-2}\right)\left(d+y_{n-2} y_{n-3}\right)\left(d+y_{n-3} y_{n-4}\right)} \\
= & \cdots \\
= & \frac{e}{d+y_{n-1} y_{n-2}}+\frac{y_{n} e}{\left(d+y_{n-1} y_{n-2}\right)\left(d+y_{n-2} y_{n-3}\right)} \\
& +\cdots+\frac{y_{n} y_{n-1} \cdots y_{1} e}{\left(d+y_{n-1} y_{n-2}\right) \cdots\left(d+y_{0} y_{-1}\right)\left(d+y_{-1} y_{-2}\right)} \\
& +\frac{y_{n} y_{n-1} \cdots y_{1} y_{0}^{2}}{\left(d+y_{n-1} y_{n-2}\right) \cdots\left(d+y_{0} y_{-1}\right)\left(d+y_{-1} y_{-2}\right)} .
\end{aligned}
$$

Thus, in view of the inequality $a^{2}+b^{2} \geq 2 a b$, we see that

$$
\begin{aligned}
y_{n+1} \leq & \frac{e}{2 \sqrt{d y_{n-1} y_{n-2}}}+\frac{y_{n} e}{4 d \sqrt{y_{n-1} y_{n-2}^{2} y_{n-3}}} \\
& +\frac{y_{n} y_{n-1} e}{8 d \sqrt{y_{n-1} y_{n-2}^{2} y_{n-3}^{2} y_{n-4} d}}+\frac{y_{n} y_{n-1} y_{n-2} e}{16 d^{2} \sqrt{y_{n-1} y_{n-2}^{2} y_{n-3}^{2} y_{n-4}^{3} y_{n-5}}} \\
& +\cdots+\frac{y_{n} y_{n-1} \cdots y_{1} e}{2^{n+1} d^{(n+1) / 2} \sqrt{y_{n-1} y_{n-2}^{2} y_{n-3}^{2} \cdots y_{0}^{2} y_{-1}^{2} y_{-2}}} \\
& +\frac{y_{n} y_{n-1} \cdots y_{1} y_{0}^{2}}{2^{n+1} d^{(n+1) / 2} \sqrt{y_{n-1} y_{n-2}^{2} \cdots y_{0}^{2} y_{-1}^{2} y_{-2}}} \\
\leq & \frac{e H^{2}}{2 \sqrt{d}}+\frac{M^{2} e H^{4}}{4 d}+\frac{M^{2} e H^{6}}{8 d \sqrt{d}}+\frac{M^{2} e H^{6}}{16 d^{2}} \\
& +\cdots+\frac{M^{2} e H^{6}}{2^{n+1} d^{(n+1) / 2}}+\frac{M^{3} H^{4}}{2^{n+1} d^{(n+1) / 2}} .
\end{aligned}
$$

Since the infinite series

$$
\sum_{n=1}^{\infty} \frac{1}{2^{n} d^{n / 2}}
$$

is convergent when $d>1 / 4$ in view of the ratio test, we see that $\lim _{n \rightarrow \infty} y_{n}=L \in[0, \infty)$ as desired. Taking limits on both sides of (2.5), we see that

$$
L=\frac{e+L^{2}}{d+L^{2}}
$$

or

$$
L^{3}-L^{2}+d L-e=0 .
$$

It is possible to find necessary and sufficient conditions such that this polynomial equation has a unique positive root. This can be done since third order polynomial equation can be solved exactly. Here, however, we will only give an example. For instance, when $d=1$ and $e=1$, the corresponding polynomial equation has the roots $1, i,-i$. Thus the corresponding solution sequence $\left\{y_{n}\right\}$ converges to 1 irrespective of the values of the positive initial conditions. 
We remark that the rational recursive sequence defined by

$$
x_{n+1}=\frac{a+b x_{n}^{2}}{c+x_{n-1} x_{n-2}}, \quad a, b, c>0 ; n=0,1,2, \ldots,
$$

can be transformed into (2.5) by writing it in the form

$$
\frac{x_{n+1}}{b}=\frac{\frac{a}{b^{3}}+\frac{x^{2}}{b^{2}}}{\frac{c}{b^{2}}+\frac{x_{n-1}}{b} \frac{x_{n-2}}{b}}, \quad n=0,1,2, \ldots
$$

The same idea can be empolyed for obtaining a different result.

Theorem 2. In addition to the assumptions in Theorem 1, suppose further that

$$
\begin{gathered}
f(x)=a+x h(x), \quad 0<x<\infty \\
g(x, y) \leq u(x) v(x), \quad 0 \leq x, y<\infty
\end{gathered}
$$

and

$$
h(x) u(x) v(x) \leq \delta<1, \quad 0 \leq x<\infty,
$$

for some positive nondecreasing function $h$ defined on $[0, \infty)$, and positive nonincreasing functions $u$ and $v$ defined on $[0, \infty)$. Then a real sequence in $\Omega$ tends to one of the positive equilibrium points of (1.1).

Proof. Let $\left\{x_{n}\right\}_{n=-2}^{\infty}$ be a real sequence in $\Omega$. Then by Theorem $1,\left\{x_{n}\right\}$ is a sequence bounded between 0 and a positive number $M$. For $n \geq 0$,

$$
\begin{aligned}
x_{n+1}= & f\left(x_{n}\right) g\left(x_{n-1}, x_{n-2}\right) \\
= & \left(a+x_{n} h\left(x_{n}\right)\right) g\left(x_{n-1}, x_{n-2}\right) \\
= & a g\left(x_{n-1}, x_{n-2}\right)+\left(a+x_{n-1} h\left(x_{n-1}\right)\right) g\left(x_{n-2}, x_{n-3}\right) h\left(x_{n}\right) g\left(x_{n-1}, x_{n-2}\right) \\
= & a g\left(x_{n-1}, x_{n-2}\right)\left(1+h\left(x_{n}\right) g\left(x_{n-2}, x_{n-3}\right)\right) \\
& +a g\left(x_{n-3}, x_{n-4}\right) h\left(x_{n}\right) h\left(x_{n-1}\right) g\left(x_{n-1}, x_{n-2}\right) g\left(x_{n-2}, x_{n-3}\right) \\
& +\cdots+a h\left(x_{n}\right) h\left(x_{n-1} \cdots h\left(x_{1}\right) g\left(x_{-1}, x_{-2}\right) g\left(x_{0}, x_{-1}\right) \cdots g\left(x_{n-1}, x_{n-2}\right)\right. \\
& +x_{0} h\left(x_{n}\right) h\left(x_{n-1}\right) \cdots h\left(x_{1}\right) h\left(x_{0}\right) g\left(x_{-1}, x_{-2}\right) g\left(x_{0}, x_{-1}\right) \cdots g\left(x_{n-1}, x_{n-2}\right) .
\end{aligned}
$$

hence, in view of our assumptions,

$$
\begin{aligned}
x_{n-1}= & a u\left(x_{n-1}\right) v\left(x_{n-2}\right)+a h\left(x_{n}\right) u\left(x_{n-1}\right) u\left(x_{n-2}\right) v\left(x_{n-2}\right) v\left(x_{n-3}\right) \\
& +a h\left(x_{n}\right) h\left(x_{n-1}\right) u\left(x_{n-1}\right) u\left(x_{n-2}\right) u\left(x_{n-3}\right) v\left(x_{n-2}\right) v\left(x_{n-3}\right) v\left(x_{n-4}\right) \\
& +a h\left(x_{n}\right) h\left(x_{n-1}\right) h\left(x_{n-2}\right) u\left(x_{n-1}\right) u\left(x_{n-2}\right) \\
& \times u\left(x_{n-3}\right) u\left(x_{n-4}\right) v\left(x_{n-2}\right) v\left(x_{n-3}\right) v\left(x_{n-4}\right) v\left(x_{n-5}\right) \\
& +\cdots \\
& +a h\left(x_{n}\right) h\left(x_{n-1}\right) \cdots h\left(x_{1}\right) u\left(x_{-1}\right) v\left(x_{-2}\right) \cdots
\end{aligned}
$$




$$
\begin{aligned}
& u\left(x_{0}\right) u\left(x_{-1}\right) v\left(x_{n-2}\right) \cdots v\left(x_{0}\right) v\left(x_{-1}\right) v\left(x_{-2}\right) \\
+ & x_{0} h\left(x_{n}\right) h\left(x_{n-1}\right) \cdots h\left(x_{0}\right) u\left(x_{n-1}\right) u\left(x_{n-2}\right) \cdots \\
& u\left(x_{0}\right) \dot{u}\left(x_{-1}\right) v\left(x_{n-2}\right) \cdots v\left(x_{-1}\right) v\left(x_{-2}\right) \\
\leq & a u\left(x_{n-1}\right) v\left(x_{n-2}\right)+a h\left(x_{n}\right) u\left(x_{n-1}\right) u\left(x_{n-2}\right) v\left(x_{n-2}\right) v\left(x_{n-3}\right) \\
+ & a h\left(x_{n}\right) h\left(x_{n-1}\right) u\left(x_{n-1}\right) u\left(x_{n-2}\right) u\left(x_{n-3}\right) v\left(x_{n-2}\right) v\left(x_{n-3}\right) v\left(x_{n-4}\right) \\
+ & a h\left(x_{n}\right) h\left(x_{n-1}\right) u\left(x_{n-1}\right) u\left(x_{n-3}\right) u\left(x_{n-4}\right) v\left(x_{n-3}\right) v\left(x_{n-4}\right) v\left(x_{n-5}\right) \delta \\
+ & \cdots \\
+ & a h\left(x_{n}\right)+h\left(x_{n-1}\right) u\left(x_{n-1}\right) u\left(x_{-1}\right) v\left(x_{-1}\right) v\left(x_{-2}\right) u\left(x_{0}\right) v\left(x_{0}\right) \delta^{n-2} \\
+ & x_{0} h\left(x_{n}\right) h\left(x_{n-1}\right) u\left(x_{n-1}\right) u\left(x_{-1}\right) v\left(x_{-1}\right) v\left(x_{-2}\right) \delta^{n-1} .
\end{aligned}
$$

By means of the monotonicity assumptions on the funtions $h, u$ and $v$, we see further that

$$
\begin{aligned}
x_{n+1} \leq & a u(0) v(0)+a h(M) u^{2}(0) v^{2}(0) \\
& +a h^{2}(M) u^{3}(0) v^{3}(0) \\
& +a h^{2}(M) u^{3}(0) v^{3}(0) \delta \\
& +\cdots \\
& +a h^{2}(M) u^{3}(0) v^{3}(0) \delta^{n-2} \\
& +x_{0} h^{2}(M) u^{2}(0) v^{2}(0) \delta^{n-1}
\end{aligned}
$$

Since $1+\delta+\cdots+\delta^{n}+\cdots$ converges when $\delta<1$, we see that $\left\{x_{n}\right\}$ converges to a nonnegative number. By taking limits on both sides of (1.1), we see that $\left\{x_{n}\right\}$ converges to one of the positive equilibrium points of (1.1). The proof is complete.

\section{References}

[1] V. L. Kocic and G. Ladas, Global asymptotic behavior of nonlinear difference equations of higher order with applications, Kluwer Academic Publishers, Dordrecht, 1993.

[2] E. Camouzis, E. A. Grove, G. Ladas and V. L. Kocic, Monotone unstable solutions of difference equations and conditions for boundedness, J. Difference Eq. Appl. 1(1995), 1744.

[3] L. T. Li, Q. S. Qiu and Z. Yu, Permanence of $x_{n+1}=x_{n}^{2} f\left(x_{n}\right) g\left(x_{n-1}\right)$, J. Difference Eq. Appl. 2(1996), 333-338.

[4] L. T. Li, Global asymptotic stability of $x_{n+1}=F\left(x_{n}\right) g\left(x_{n-1}\right)$, Ann. Diff. Eq., to appear.

Department of Mathematics, Wuyi Univeristy, Jiangmen, Gauagdong, 529020, P. R. China.

Department of Mathematics, Tshing Hua University, Hsinchu, Taiwan 30043, R. O. C. 